\title{
NEUROTRANSMITTERS FOR MATH BRAIN: A NEW FINDING
}

\author{
Taimoor Hassan ${ }^{1,2}$ \\ ${ }^{1}$ School of Pharmacy \& School of Medicine, Changzhou University, Jiangsu, China, \\ ${ }^{2}$ Department of Health Professional Technologies, The University of Lahore, Pakistan
}

\section{What are Neurotransmitters?}

Neurotransmitters are the body's chemical transmitters. Their task is to send nerve cell messages to target cells. ${ }^{1}$ The target cells might be found in muscles or glands and other nerves. In order to control many of the processes needed for the brain, it needs neurotransmitters, including heart rate, respiration, circadian rhythms, digestion etc. $^{2}$ The nervous system regulates the organs and psychological and physical operations of the individual. ${ }^{3}$ The neurotransmitters of nerve cells, also known as neural messengers, have a substantial function. The cells of the nerves fire impulses. ${ }^{4}$ This is done by the release of neurotransmitters; chemical substances that relay signals to other cells. Each neurotransmitter binds to another receptor, for instance; dopamine molecules attach to the receptors of dopamine. ${ }^{5}$ This initiates action in the target cells when they are coupled. The body eliminates or recycles them after neurotransmitters send their messages. ${ }^{2}$

\section{Are they GABA and Glutamate?}

- An individual's mathematical aptitude may be associated with the amounts of gamma-aminobutyric acid (GABA) and glutamate in the brain, indicated by research. ${ }^{3}$

- To identify this, researchers tested and linked levels of these neurotransmitters with test results in children and adults.

- Children who were strong in mathematics prob- ably had greater GABA levels in their brains and lower glutamate levels.

- In the meanwhile, the opposite for adults was true: lower levels of GABA and higher glutamate represented increased mathematical skills.

- The results imply that quantities of neurotransmitters in the brain might forecast future mathematical skills. ${ }^{3}$

The study, led by cognitive neuroscience Professors, George Zacharopoulos and Roi Cohen Kadosh of Oxford University in the United Kingdom, examined GABAs and brain glutamate levels to see if those neurotransmitters might predict future mathematical abilities. While talking to Medical News Today, Dr. Kadosh said: "We have focused on GABA and glutamate since it is thought that these neurotransmitters are main stakeholders in cognition, neuroplasticity, and learning. We chose mathematical ability since developing a real gift takes years (if at all). It is a complex cognitive ability. This confluence made the experiment highly interesting since we could show how GABA and glutamate contribute in a sophisticated cognitive skill that takes years to develop." 4 Prof. Kadosh and his colleagues observed not just a connection between these neurotransmitters, but also the changes in neurotransmitters as children grow into adults.

\section{What are their Study Findings?}

In the study, 255 individuals aged six to university level were analyzed and assessed for their performance

KEY WORDS: Gamma-Aminobutyric Acid; Glutamic Acid; Neurotransmitter Agents.

Cite as: Hassan T. Neurotransmitters for math brain: a new finding [editorial]. Gomal J Med Sci 2021 Oct-Dec; 19(4):125-6. https://doi.org/10.46903/gjms/19.04.954

\section{Corresponding Author:}

Taimoor Hassan

Post-graduate Scholar,

School of Pharmacy \& School of Medicine,

Changzhou University, Jiangsu, China

E-mail: taimoorhassan408.th@gmail.com

Date Submitted:

05-08-2021

Date Revised:

Date Accepted:
$17-09-2021$

24-09-2021 by two mathematical exams over a period of 1.5 years. The test findings were then linked with the GABA and glutamate in their brain. Those youngsters with greater amounts of GABA - it is present in left intraparietal sulcus (IPS), a region of brain - got a better score in mathematical testing. Conversely, the high glutamate test results in the IPS were lower. However, the scientists observed the reverse in adults. Those with high glutamate levels in their brains had excellent arithmetic test values while 
those with high GABA levels had lower score. After evaluating the subjects twice and 1.5 years apart, the researchers discovered that adults with reduced GABA performed well on the first arithmetic exam and also performed well on the second test. ${ }^{3,6}$

\section{What does exactly findings mean?}

The longitudinal approach used by the scientists aided them in forecasting future mathematical aptitude. The research also indicates that GABA and glutamate levels in the brain change later in puberty. This suggests that the role of these neurotransmitters vary as a person grows. "The most striking discovery was that the link between GABA and glutamate and mathematical competence was reversed from infancy to maturity," said Dr. Roi Kohen. It demonstrates that the relationship between GABA and glutamate and skill acquisition/ ability differs with developmental stage and is age dependent.

Dr. Santosh Kesari, PhD and neuroscientist at the Providence St John's Health Center in Santa Monica, California commented on the aforementioned study and said "we do know that things change during brain development, and the sensitivity of a brain area to a certain neurotransmitter may be altered as the brain develops and matures. So, even if it is the same transmitter, such as GABA or glutamate, the impacts on how those neurotransmitters may operate or affect the brain may change earlier in development versus later in development." ${ }^{3}$

\section{What is future potential of this study?}

Prof. Kadosh advises care when using this data for real-world applications. Nonetheless, it has created a huge opportunity. He stated that: "Because his study only included healthy children, adolescents, and adults, it was too early to discuss clinical implications at this point."

"It would be fascinating to observe how these results appear in people who have learning challenges, and whether manipulating GABA and/ or glutamate may enhance learning and education," he added. ${ }^{3,4}$

Meanwhile, Dr. Kesari stated that there is an age dependence that might impact on how we teach or develop mathematical ability in both young and old children. The next step in this research might be to develop better approaches for teaching and assisting kids in learning arithmetic, as well as to investigate the prospect of developing learning interventions to improve cognition. ${ }^{5}$

"We are developing neurostimulation techniques to enhance learning and cognition. We investigate its use in clinical (children and adults) and non-clinical groups (only adults). Early literature has shown that various neurostimulation techniques can alter GABA and/ or glutamate levels. We believe that this study would pave the path for those people who are in the need of improving their learning and cognition abilities." 3

\section{REFERENCES}

1. Wang F, Yang J, Pan F, Ho RC, Huang JH. Neurotransmitters and emotion [editorial]. Front Psychol 2020 Jan 29;11:21. https://doi.org/10.3389/ fpsyg.2020.00021

2. Tian W, Chen S. Neurotransmitters, cell types, and circuit mechanisms of motor skill learning and clinical applications. Front Neurol 2021 Feb 25;12:616820. https://doi.org/10.3389/ fneur.2021.616820

3. Sakay NY. The math brain: Scientists find two predictive neurotransmitters. Medical News Today [Online]. [cited 2021 Aug 3]. Available at: https://www.medicalnewstoday.com/articles/ the-math-brain-scientists-find-2-predictive-neurotransmitters

4. Handra C, Coman OA, Coman LA, Enache T, Stoleru S, Sorescu AM, et al. The connection between different neurotransmitters involved in cognitive processes. Farmacia 2019 Mar 1;67(2):193201. https://doi.org/10.31925/farmacia.2019.2.1

5. Altaib H, Nakamura K, Abe M, Badr Y, Yanase E, Nomura I, et al. Differences in the concentration of the fecal neurotransmitters GABA and glutamate are associated with microbial composition among healthy human subjects. Microorganisms 2021 Feb;9(2):378-84. https://doi.org/10.3390/ microorganisms9020378

6. Zacharopoulos G, Sella F, Cohen Kadosh K, Hartwright C, Emir U, Cohen Kadosh R. Predicting learning and achievement using GABA and glutamate concentrations in human development. PLoS Biol 2021 Jul 22;19(7):e3001325. https:// doi.org/10.1371/journal.pbio.3001325

CONFLICT OF INTEREST

Authors declare no conflict of interest.

GRANT SUPPORT AND FINANCIAL DISCLOSURE None declared.

Copyright (C) 2021. Taimoor Hassan. This is an Open Access article distributed under the terms of the Creative Commons Attribution-NonCommercial 4.0 International License, which permits unrestricted use, distribution \& reproduction in any medium provided that original work is cited properly. 